\title{
PROBLEM FOR SOLUTION
}

P. 162. Let $\mathrm{G}$ be a finite abelian group, written additively, and $S$ a subset of $G . S$ is said to be a sum-free set in $G$ if $(S+S) \cap S=\phi$. Let $\lambda(G)$ denote the largest possible order of a sum-free set in $G$.

For which abelian groups $G$ does there exist a sum-free set $S$ such that (i) $|S|=\lambda(G)$

and (ii) $|S+S|=\frac{\lambda(G)[\lambda(G)+1]}{2}$ ?

A.P. Street, University of Alberta

\section{SOLUTIONS}

P. 154. Let $\mathrm{n}$ identical weighted coins, each falling heads with probability $x$, be tossed, and let $p_{i}(x)$ be the probability that exactly $i$ of them fall heads. Evaluate

$$
\begin{aligned}
& f_{n}=\min _{0 \leq x \leq 1} \quad \max _{i=1, \ldots, n} \quad p_{i}(x) \\
& \text { W. Moser, McGill University }
\end{aligned}
$$

\section{Solution by D. Ž. Djoković, University of Waterloo}

$$
\text { Let } f_{n}(x)=\max _{i=0,1, \ldots, n} p_{i}(x) \text {. }
$$

Since

$$
p_{i}(x)=\left(\begin{array}{l}
n \\
i
\end{array}\right) x^{i}(1-x)^{n-i}
$$

and

$$
\frac{p_{i}(x)}{p_{i+1}(x)}=\frac{i+1}{n-1} \cdot \frac{1-x}{x} \quad(i=0,1, \ldots, n-1)
$$


we infer that

$$
f_{n}(x)=\left(\begin{array}{l}
n \\
i
\end{array}\right) x^{i}(1-x)^{n-i}, \frac{i}{n+1} \leq x \leq \frac{i+1}{n+1}
$$

for each $i=0,1, \ldots, n$. We see that $f_{n}(x)$ is decreasing in $\left(0, \frac{1}{n+1}\right)$ and increasing in $\left(\frac{n}{n+1}, 1\right)$. In the interval $\left(\frac{i}{n+1}, \frac{i+1}{n+1}\right)(i=1, \ldots, n-1)$ it is increasing for $\frac{i}{n+1}<x<\frac{i}{n}$ and decreasing for $\frac{i}{n}<x<\frac{i+1}{n+1}$. Ther efore

$$
f_{n}=\min _{i=1, \ldots, n} a_{i}
$$

where

$$
a_{i}=f_{n}\left(\frac{i}{n+1}\right)=\left({ }_{i}^{n}\right) \frac{i^{i}(n+1-i)^{n-i}}{(n+1)^{n}}
$$

We have

$$
\frac{a_{i}}{a_{i+1}}=\frac{\left(1+\frac{1}{n-i}\right)^{n-i}}{\left(1+\frac{1}{i}\right)^{i}}>1, \quad \text { if } i<\frac{n}{2} \text {. }
$$

Taking into account the symmetry $\left(a_{i}=a_{n+1-i}\right)$, we get

$$
f_{n}=\left(\begin{array}{l}
n \\
k
\end{array}\right) \frac{k^{k}(n+1-k)^{n-k}}{(n+1)^{n}}
$$

where $k$ is the integral part of $\frac{\mathrm{n}}{2}$.

Also solved by G. Letac, I. B. MacNeill, K.G. Miller, R. A. Schaufele, and the proposer. 
P. 155. If $\mathrm{a}_{1}<\mathrm{a}_{2}<\ldots<\mathrm{a}_{\mathrm{k}} \leq \mathrm{n}$ is a sequence of positive integers such that $\left[a_{i}, a_{j}\right]>n$ for all $i \neq j$, show that $\sum_{i=1}^{k} \frac{1}{a_{i}}<2$,

$\left(\left[a_{i}, a_{j}\right]\right.$ means "the least common multiple of $a_{i}$ and $\left.a_{j}{ }^{\prime \prime}\right)$.

\section{Anonymous}

P. Erdols and A. Makowski have pointed out that this problem was posed for solution in the Amer. Math. Monthly, 56 (1949) p. 637, by Erdös. A solution with a better constant than 2, by R. Lehman appeared ibid., 58 (1951), p 345. Finally, A. Schinzel and G. Szekeres, Acta Sci. Math. (= Acta Szeged) 20 (1959) pp. 221-229, prove that the sum considered is $\leq 31 / 30$ and that this constant is best possible. 